\title{
Near-field radiative heat transfer between one-dimensional magneto-photonic crystals
}

\author{
E. Moncada-Villa ${ }^{1}$ and J. C. Cuevas ${ }^{2}$ \\ ${ }^{1}$ Escuela de Física, Universidad Pedagógica y Tecnológica de Colombia, \\ Avenida Central del Norte 39-115, Tunja, Colombia and \\ ${ }^{2}$ Departamento de Física Teórica de la Materia Condensada and Condensed Matter Physics Center (IFIMAC), \\ Universidad Autónoma de Madrid, E-28049 Madrid, Spain
}

(Dated: October 7, 2020)

\begin{abstract}
We present a theoretical study of the effect of an external dc magnetic field in the near-field radiative heat transfer between two one-dimensional magneto-photonic crystals with unit cells comprising a magneto-optical layer made of $n$-doped $\mathrm{InSb}$ and a dielectric layer. We find that in absence of an external field, and depending on the gap size, the radiative heat transfer between these multilayer structures can be larger or smaller than that of the case of two InSb infinite plates. On the other hand, when an external magnetic field is applied, the near-field radiative heat transfer is reduced as a consequence of the suppression of hybridized surface polariton waves that are supported for transverse magnetic polarized light. We show that such reduction is exclusively due to the appearance of magnetic-field induced hyperbolic modes, and not to the polarization conversion in this magneto-optical system.
\end{abstract}

\section{INTRODUCTION}

In recent years we have witnessed a true revolution in the field of thermal radiation [1]. This has been mainly triggered off by experimental advances that, in particular, have made possible to confirm the long-standing prediction that the limit set by Stefan-Boltzmann's law for the radiative heat transfer between two bodies can be largely overcome by bringing them sufficiently close [4. In the near-field regime, i.e., when the separation between two bodies is smaller than the thermal wavelength $\lambda_{\mathrm{Th}}(\sim 10 \mu \mathrm{m}$ at room temperature), they can also exchange radiative heat via evanescent waves (or photon tunneling), which are not taken into account in the derivation of Stefan-Boltzmann's law. This additional contribution can completely dominate the near-field radiative heat transfer (NFRHT) and, in turn, can lead to overcome the Stefan-Boltzmann's limit (or blackbody limit) by orders of magnitude for sufficiently small gaps. There is by now an overwhelming experimental evidence of this fact that has obtained in a great variety of systems and using many different materials [5-29]. From a fundamental point of view, these experiments have also helped to firmly establish the theory of fluctuational electrodynamics 30, 31, which has become the standard model for the description of NFRHT. From a more applied point of view, NFRHT may have an important impact in different technologies that make use of thermal radiation, see Refs. [1] 3] and references therein.

The basic physical mechanisms underlying NFRHT are relatively well-understood by now. In this sense, a good part of the efforts of the theory community in the field of thermal radiation focuses now on finding strategies to actively control NFRHT with the hope to develop novel thermal functional devices. Many interesting ideas have been put forward in recent years, and the interested reader can consult the recent reviews of Refs. 1 1 3. One of the most attractive and promising ideas is the use an external magnetic field to control the
NFRHT between magneto-optical (MO) materials, which we proposed some years ago in Ref. 32. In that work, we showed that the NFRHT between two parallel plates made of doped semiconductors can be substantially altered by the application of a static magnetic field. This work paved the way for the prediction of a plethora of thermomagnetic effects. Thus, for instance, it has been predicted that the lack of reciprocity in MO systems can lead to novel phenomena such as a near-field thermal Hall effect 33 or the existence of a persistent heat current 34. It has also been theoretically demonstrated that MO systems under a static magnetic field can exhibit many phenomena that are the near-field thermal analogues of basic transport effects in the field of spintronics such as giant thermal magnetoresistance 35] or anisotropic thermal magnetoresistance 36, 37. Many of these phenomena have been reviewed in Refs. [3, 38.

Most theoretical studies of the effect of a magnetic field in the NFRHT between MO systems have shown that the field tend to reduce the NFRHT and only a modest enhancement was found in Ref. 37 in a very asymmetric situation. The reason for this reduction in extended systems (like infinite parallel plates) is that a magnetic field induces the appearance of hyperbolic modes that replace the surface modes, both surface plasmon polaritons (SPPs) and surface phonon polaritions (SPhPs), that dominate the NFRHT in these systems in the absence of magnetic field [32, 39]. These hyperbolic modes, in spite of being propagating inside the material, are less effective than surface modes transferring the heat across the gap between two bodies. In this regard, the recent prediction put forward in Ref. [40, that an external magnetic field can greatly enhance the NFRHT between two multilayer hyperbolic metamaterials (consisting of alternating layers of a MO material and a dielectric) is certainly very interesting, albeit quite surprising. There has been quite some attention devoted to this type of multilayer systems in recent years [4148]. The main reason for this interest is that the contribution of surface modes 
at multiple interfaces can greatly enhance the NFRHT, as compared to the case of two infinite parallel plates, see e.g. Ref. [48. The conclusion that an external magnetic can enhance the NFRHT between one-dimensional (1D) magneto-photonic crystals was reached in Ref. 40. making use of an effective medium theory, a type of theory that is known to have limitations in the description of NFRHT [47 49]. In this regard, it would be highly desirable to revisit this interesting problem making use of an exact approach. This is precisely the main goal of this work.

In this paper we present a systematic theoretical study of the influence of an external magnetic field in the NFRHT between two identical 1D magneto-photonic crystals with unit cells comprising a MO layer, made of InSb, and a dielectric layer, made of either BK7 glass or $\mathrm{SiO}_{2}$, see Fig. 1(a). Our calculations are based on a scattering matrix approach for anisotropic materials that provides the exact numerical results for an arbitrary number of layers in these crystals. We find that, irrespective of the different geometrical parameters, such as gap size, thickness of the dielectric layer, etc., and irrespective the dielectric material (BK7 glass or silica), an external magnetic field always reduces the NFRHT as compared to the case of two InSb parallel plates. In particular, we find that for a field of a few Teslas the NFRHT of these layered systems can be reduced by almost a factor of 6 , as compared to the zero-field case. Moreover, we show that the physical mechanism for this drastic magnetic-field reduction is the appearance of hyperbolic modes that replace the hybrid surface polariton modes supported by these metal-dielectric multilayer structures.

The remainder of this paper is organized as follows. In Sec. II we describe the 1D magneto-photonic crystals under study, as well as the theoretical approach used for the calculation of the radiative heat transfer between them. In Sec. III], we start the discussion of our main results by analyzing the radiative heat transfer in the absence of an applied field, paying special attention to the reduction of the heat flux in an intermediate regime of the vacuum gap size, and to the enhancement of radiative heat flux in the far-field regime. Then, in Sec. IV] we analyze the effect of an external magnetic field in the NFRHT between these multilayer structures. Finally, in Sec. $\mathrm{V}$ we summarize the main results presented in this manuscript.

\section{SYSTEM UNDER STUDY AND THEORETICAL APPROACH}

The goal of this work is the theoretical description of the radiative heat transfer between two identical multilayer structures, both deposited on InSb substrates, separated by a vacuum gap of size $d$ and under the action of an external dc magnetic field of magnitude $H$ [see Fig. 1(a)]. Each multilayer comprises $N$ unit cells with a dielectric layer of either $\mathrm{SiO} 2$ or BK7 glass, of thickness $t_{d}$, and a MO layer of InSb, with thickness $t_{m}$. The external mag-
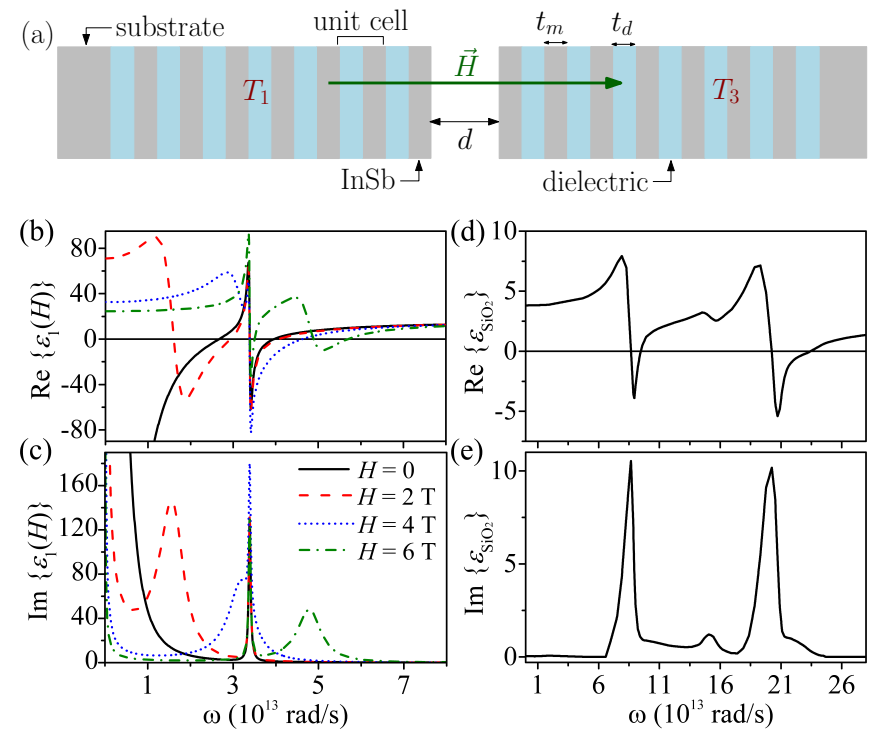

FIG. 1. (a) Schematic view of two identical periodic multilayer structures, separated by a vacuum gap of thickness $d$, each one at its corresponding temperature $T_{1}$ and $T_{3}$, and under the influence of an external dc magnetic field $H$ oriented along the stratification $(z)$ axis. The unit cells comprise a layer of InSb, with thickness $t_{m}$, and a dielectric layer such as $\mathrm{BK} 7$ or $\mathrm{SiO}_{2}$ of thickness $t_{d}$ (assumed to be $10 \mathrm{~nm}$ in all calculations). Both structures are grown on InSb substrates. In panels (b) and (c), we show, the real and imaginary part, respectively, of the diagonal element of InSb dielectric tensor for different values of the external magnetic field. In panels (d) and (e) we present the real and imaginary part, respectively, of the dielectric function of $\mathrm{SiO}_{2}$.

netic field, assumed to be parallel to the stratification axis ( $z$-axis), generates a MO activity inside the $\mathrm{InSb}$ material [50, which is described by the following dielectric tensor [51]

$$
\hat{\epsilon}=\left(\begin{array}{ccc}
\epsilon_{1} & -i \epsilon_{2} & 0 \\
i \epsilon_{2} & \epsilon_{1} & 0 \\
0 & 0 & \epsilon_{3}
\end{array}\right),
$$

where

$$
\begin{aligned}
\epsilon_{1}(H) & =\epsilon_{\infty}\left(1+\frac{\omega_{L}^{2}-\omega_{T}^{2}}{\omega_{T}^{2}-\omega^{2}-i \Gamma \omega}+\frac{\omega_{p}^{2}(\omega+i \gamma)}{\omega\left[\omega_{c}^{2}-(\omega+i \gamma)^{2}\right]}\right) \\
\epsilon_{2}(H) & =\frac{\epsilon_{\infty} \omega_{p}^{2} \omega_{c}}{\omega\left[(\omega+i \gamma)^{2}-\omega_{c}^{2}\right]}, \\
\epsilon_{3} & =\epsilon_{\infty}\left(1+\frac{\omega_{L}^{2}-\omega_{T}^{2}}{\omega_{T}^{2}-\omega^{2}-i \Gamma \omega}-\frac{\omega_{p}^{2}}{\omega(\omega+i \gamma)}\right) .
\end{aligned}
$$

Here, $\epsilon_{\infty}$ is the high-frequency dielectric constant, $\omega_{L}$ $\left(\omega_{T}\right)$ is the longitudinal (transverse) optical phonon frequency, $\omega_{p}^{2}=n e^{2} /\left(m^{*} \epsilon_{0} \epsilon_{\infty}\right)$ is the plasma frequency of free carriers of density $n$ and effective mass $m^{*}, \Gamma$ $(\gamma)$ is the phonon (free-carrier) damping constant, and $\omega_{c}=e H / m^{*}$ is the cyclotron frequency, which depends on the intensity of the external magnetic field. All the 
calculations presented in this work were done assuming the following parameter values taken from Ref. [51]: $\epsilon_{\infty}=15.7, \omega_{L}=3.62 \times 10^{13} \mathrm{rad} / \mathrm{s}, \omega_{T}=3.39 \times 10^{13}$ $\mathrm{rad} / \mathrm{s}, \Gamma=5.65 \times 10^{11} \mathrm{rad} / \mathrm{s}, \gamma=3.39 \times 10^{12} \mathrm{rad} / \mathrm{s}$, $n=1.07 \times 10^{17} \mathrm{~cm}^{-3}, \mathrm{~m}^{*} / \mathrm{m}=0.022, \omega_{p}=3.14 \times 10^{13}$ $\mathrm{rad} /$. Moreover, we set the thickness of the dielectric layers to $t_{d}=10 \mathrm{~nm}$. We present in Fig. 1 (b,c) the real and imaginary parts of the component $\epsilon_{1}(H)$ for such a choice of parameters and different values of the magnitude of the applied field. According to Eq. (2), in the absence of an external field it follows that $\epsilon_{1}(H=0)=\epsilon_{3}$. However, as can be observed in Fig. 1(b), the application of an external field leads to the appearance of frequency regions in which $\epsilon_{1}(H)$ and $\epsilon_{3}$ have opposite signs. These regions are characterized by the existence of electromagnetic modes, known as hyperbolic modes, that are propagating inside the InSb and evanescent out of it. These modes are responsible of the progressive disappearance of the SPP modes occurring below the surface plasmon frequency $\omega_{s p}=\omega_{p} / \sqrt{2}$, and of the SPhP modes occurring between the longitudinal and transverse optical frequencies $\omega_{T}$ and $\omega_{L}$, as it has been shown for infinite parallel plates and thin films made of InSb 32, 39.

In this work, we consider two possible materials for the dielectric layers. The first one is the BK7 glass, with a constant (and real) dielectric function of $\varepsilon_{d}=2.25$. The second one is $\mathrm{SiO}_{2}$, a polar material whose dielectric function has the real and imaginary parts presented in panels (d) and (e) of Fig. 1 that were taken from Ref. [52. As it is well-known, for frequencies in which the real part of this dielectric function is negative, an interface between an infinite plate of this material and vacuum can support SPhPs whose evanescent field contributes substantially to the NFRHT transfer [9, 19, 53.

As explained above, we focus here on the calculation of the radiative heat flux between two 1D magneto-photonic crystals [see Fig. 1(a)]. This calculation was performed within the framework of the fluctuational electrodynamics theory [30, 31]. In particular, we are interested in the radiative linear heat conductance per unit of area or heat transfer coefficient, $h_{N}$, between the two anisotropic multilayer systems, each one at its corresponding temperature $T_{1}$ and $T_{3}$ [see Fig. 1(a)]. This heat transfer coefficient is defined in terms of the net power per unit of area exchanged between two anisotropic layered systems, $Q_{N}$, via the relation

$$
h_{N}(T, d)=\lim _{\Delta T \rightarrow 0^{+}} \frac{Q_{N}\left(T_{1}=T+\Delta T, T_{3}=T, d\right)}{\Delta T},
$$

where [54]

$$
Q_{N}=\int_{0}^{\infty} \frac{d \omega}{2 \pi}\left[\Theta_{1}(\omega)-\Theta_{3}(\omega)\right] \int \frac{d \mathbf{k}}{(2 \pi)^{2}} \tau(\omega, \mathbf{k}, d) .
$$

In this Landauer-like expression, $\Theta_{i}(\omega)=$ $\hbar \omega /\left[\exp \left(\hbar \omega / k_{\mathrm{B}} T_{i}\right)-1\right]$ describes the energy of the thermally occupied photonic states with frequency $\omega$ and wave vector parallel to the interfaces in the multilayers $\mathbf{k}=\left(k_{x}, k_{y}\right)$. The function $\tau(\omega, \mathbf{k}, d)$ is the total transmission probability of the electromagnetic propagating waves $(|\mathbf{k}|=k<\omega / c)$, as well as evanescent ones $(k>\omega / c)$, and is expressed as [32, 54.

$$
\begin{aligned}
& \tau(\omega, \mathbf{k}, d)= \\
& \left\{\begin{array}{ll}
\operatorname{Tr}\left\{\left[\hat{1}-\hat{\mathcal{R}}_{21} \hat{\mathcal{R}}_{21}^{\dagger}\right] \hat{\mathcal{D}}^{\dagger}\left[\hat{1}-\hat{\mathcal{R}}_{23}^{\dagger} \hat{\mathcal{R}}_{23}\right] \hat{\mathcal{D}}\right\}, & k<\omega / c \\
\operatorname{Tr}\left\{\left[\hat{\mathcal{R}}_{21}-\hat{\mathcal{R}}_{21}^{\dagger}\right] \hat{\mathcal{D}}^{\dagger}\left[\hat{\mathcal{R}}_{23}^{\dagger}-\hat{\mathcal{R}}_{23}\right] \hat{\mathcal{D}}\right\} e^{-2\left|q_{2}\right| d}, & k>\omega / c
\end{array} .\right.
\end{aligned}
$$

Here, $q_{2}=\sqrt{\omega^{2} / c^{2}-k^{2}}$ is the $z$-component of the wave vector in the vacuum gap, $c$ is the velocity of light in vacuum and $\hat{\mathcal{D}}=\left[\hat{1}-\hat{\mathcal{R}}_{21} \hat{\mathcal{R}}_{23} e^{2 i q_{2} d}\right]^{-1}$ describes the usual Fabry-Pérot-like denominator resulting from the multiple scattering in the vacuum gap between the two multilayered systems. The $2 \times 2$ matrices $\hat{\mathcal{R}}_{i j}$ are the reflections matrices characterizing the two interfaces at both sides of the gap, and contain the information of the multiple scattering processes taking place inside each structure. These matrices have the following generic structure

$$
\hat{\mathcal{R}}_{i j}=\left(\begin{array}{cc}
r_{i j}^{s, s} & r_{i j}^{s, p} \\
r_{i j}^{p, s} & r_{i j}^{p, p}
\end{array}\right),
$$

where $r_{i j}^{\alpha, \beta}$ with $\alpha, \beta=s, p$ (or TE, TM) is the reflection amplitude for the scattering of an incoming $\alpha$-polarized plane wave into an outgoing $\beta$-polarized wave. In practice, we compute numerically the different reflection matrices appearing in Eq. (5) by using the scattering-matrix approach for anisotropic multilayer systems that is described in Refs. [32, 55]. It is worth remarking that the approach used in this work, contrary to the effective medium theory [40, 56, provides the exact numerical results for this problem and it can be applied for an arbitrary number of unit cells in the 1D crystals. Let us also say that all the results presented in the work for the heat transfer coefficient were obtained for room temperature $(T=300 \mathrm{~K})$.

\section{ZERO-FIELD RADIATIVE HEAT TRANSFER}

We start the discussion of our results by first considering the radiative heat transfer between the multilayer systems of Fig. 1(a) in the absence of an external magnetic field. Radiative heat transfer in the near-field regime for this kind of systems, in absence of an external magnetic field, has already been reported in the literature [47, 48]. In these works, it was shown that it is possible to enhance the NFRHT exchanged by two periodic multilayers, $h_{N}$, relative to the heat transfer between two infinite plates $h_{\text {bulk. }}$. Such an enhancement is a consequence of the hybridization of SPP and SPhP modes with the Bloch modes resulting from the translation symmetry in a periodic structure. However, no much attention has been paid to the radiative heat transfer in the intermediate and far-field regimes. With this in mind, we start by presenting in Fig. 2(a-c) the heat transfer coefficient for 

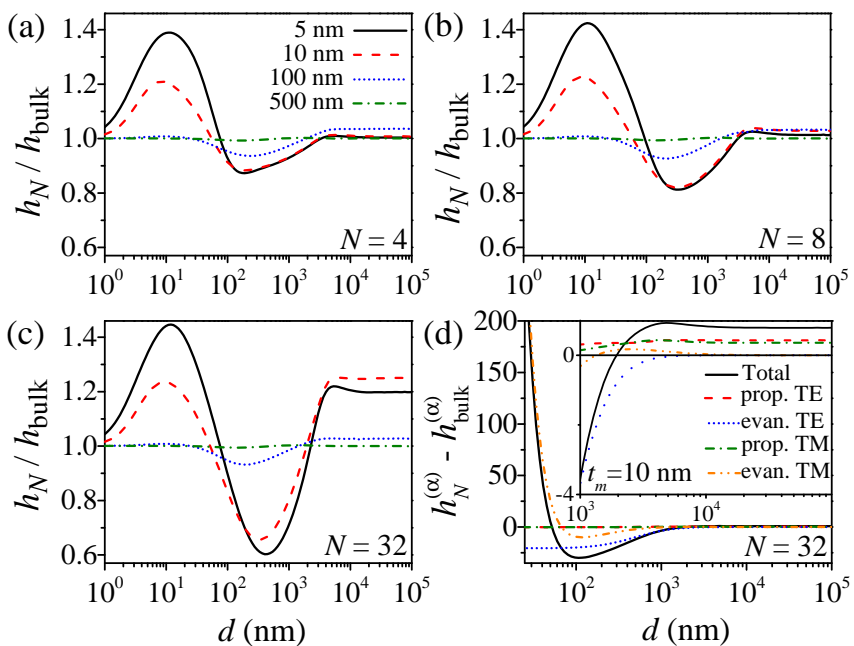

FIG. 2. (a-c) Ratio between the zero-field heat transfer coefficient of two multilayers, $h_{N}$, and the heat transfer coefficient of two semi-infinite plates of InSb, $h_{\mathrm{bulk}}$, as a function of the gap size $d$. Panels (a), (b) and (c), correspond to $N=4$, $N=8$ and $N=32$ unit cells, respectively, and the different lines in each graph correspond to different values of thickness of the MO layers of InSb: $5 \mathrm{~nm}$ (solid line), $10 \mathrm{~nm}$ (dashed line), $100 \mathrm{~nm}$ (dotted line), and $500 \mathrm{~nm}$ (dash dotted line). The dielectric material is BK7 and its layers have a thickness of $10 \mathrm{~nm}$. (d) Gap dependence of the differences $h_{N}^{(\alpha)}-h_{\text {bulk }}^{(\alpha)}$, where $\alpha$ stands for total, propagating and evanescent TE or TM, for $N=32$ unit cells, and $t_{m}=10 \mathrm{~nm}$ [i.e., for the dashed line in panel (c)].

the two multilayer structures, $h_{N}$, as a function of the gap size $d$, normalized to the heat transfer coefficient for two InSb infinite plates, $h_{\text {bulk }}$, for three different values of the number of unit cells in each structure, $N$, and for several values of the thickness of the InSb layers, $t_{m}$. In this case we assumed that the dielectric layers were made of BK7 glass. For gap sizes between $10 \mathrm{~nm}$ to $80 \mathrm{~nm}$ we observe an enhancement of the NFRHT, which becomes more prominent as the thickness of the InSb layers is reduced and it reaches a maximum at $d \sim 10 \mathrm{~nm}$. This is in qualitative agreement with what it has been reported in literature for layered structures with metal/vacuum unit cells 48. However, for gap sizes larger than about $80 \mathrm{~nm}$, this enhancement disappears up to gaps on the order of $2200 \mathrm{~nm}$ and the radiative heat flux between the multilayer systems is actually smaller than in the case of two infinite parallel plates of InSb. Beyond gap sizes of about $2200 \mathrm{~nm}$, an in particular in the far-field regime, the radiative heat flux between multilayered stacks is again larger than the corresponding one for two InSb parallel plates.

In order to understand these new features for gap sizes above $80 \mathrm{~nm}$, we present in Fig. 2(d) the difference between the total heat transfer coefficients $h_{N}$ and $h_{\mathrm{bulk}}$, and between each one of their separate contributions (evanescent and propagating waves for TE and TM polarizations). This is done for the case represented by a dashed line in panel (c). As it can be seen, the enhancement in the near-field regime $(d \leq 80 \mathrm{~nm})$ is entirely due to the contribution of the TM evanescent modes associated to the SPP and SPhPs modes. We also see that the decrease of the heat flux $h_{N}$ relative to $h_{\text {bulk }}$ for gap sizes between $80 \mathrm{~nm}$ and $2200 \mathrm{~nm}$ is due to the decrease of both TE and TM evanescent contributions to the total flux. To provide some physical insight for the decrease of these contributions, we present in Fig. 3 the comparison between the transmission probabilities corresponding to the multilayer configuration and the corresponding ones for two infinite InSb plates, for TE and TM polarizations and for two different values of the gap size. We observe from panels (a) and (e) that the electromagnetic modes on the right of the vacuum light line $\omega=c k$ and on the left of the InSb light line $\omega=c k / \operatorname{Re}\left(\epsilon_{1}^{1 / 2}\right)$, i.e., the frustrated TE evanescent modes, are more probable for the case of two infinite plates than for the multilayer system. This change is due to the fact that the outermost layer in the stratified media has less propagating modes involved in total internal reflection at the interface with the vacuum gap. This decrease in the contribution of frustrated TE modes is indeed evident in Fig. 4(a), where we have plotted the spectral heat transfer contribution, $h_{\omega}$, corresponding to the transmission probabilities of panels (a) and (e) in Fig. 3. Let us clarify that this spectral heat transfer coefficient is defined as the heat transfer coefficient per unit of frequency. Concerning the TM evanescent waves, we can see from panels (b) and (f) of Fig. 3 that transmission for the multilayer structures exhibits a higher number of surface states as a consequence of the hybridization of SPP and SPhP modes with Bloch modes. However, these hybridized modes have smaller values of the parallel component of the wave vector $k$ than in the case of two infinite plates. The reason for this is that as the gap size increases, the evanescent field of deepest layers in one structure cannot reach the vacuum gap and penetrate into the first layer of opposite structure. Then, as it can be observed in Fig. 4(b), the corresponding spectral heat transfer coefficient has broader, but smaller central peaks than in the configuration of two infinite plates.

We end this section by briefly discussing the increase of the far-field radiative heat transfer, see inset in Fig. 2. It is clear that such an increase originates from both TE and TM propagating modes, which in turn is a consequence of the enhanced intensity of Fabry-Pérot resonances inside all the $2 N$ layers of each multilayer system, as can be seen upon comparing panels (c) and (g) $[(\mathrm{d})$ and (i) ] for TE (TM) polarized waves, and in their corresponding spectral heat transfer coefficients in Fig. 4(c) [4(d)].

\section{MAGNETIC FIELD EFFECT ON THE RADIATIVE HEAT TRANSFER}

We turn now to discuss how the magnetic field affects the radiative heat transfer in the system under study. In 

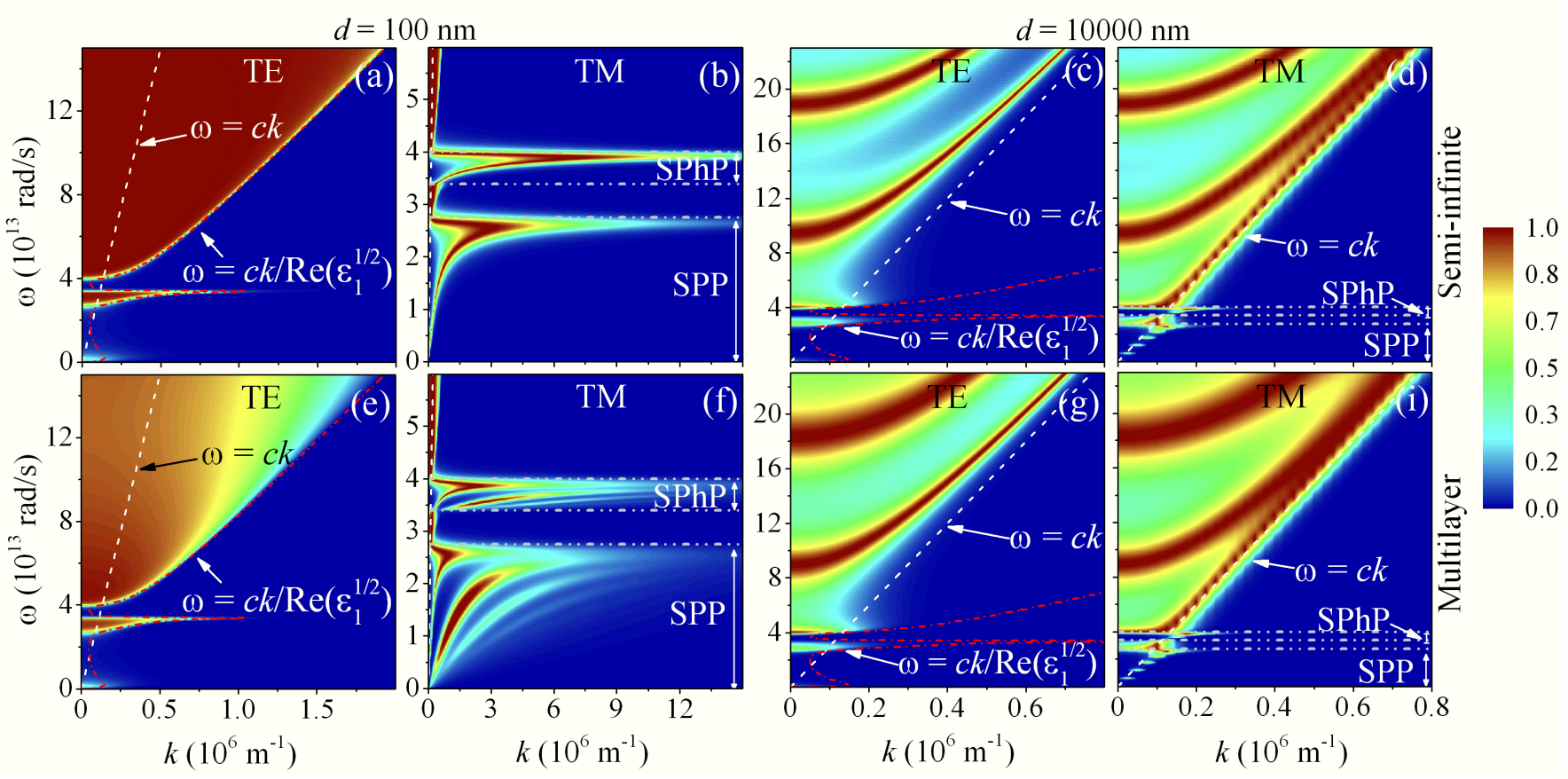

FIG. 3. Transmission coefficient for s-polarized (TE) [panels (a), (c), (e) and (g)] and p-polarized (TM) [panels (b), (d), (f) and (i)] waves, as a function of the magnitude of the parallel wave vector and frequency, in absence of an external magnetic field. Panels (a), (b), (e) and (f), correspond to a gap size $d=100 \mathrm{~nm}$, whereas the rest to a gap size of $d=10000 \mathrm{~nm}$. The white dashed lines indicate the light line in vacuum, $\omega=c k$, while the red dashed-dotted lines in panels (a), (c), (e) and (g) correspond to the light line inside $\operatorname{InSb}, \omega=c k / \operatorname{Re}\left(\epsilon_{1}^{1 / 2}\right)$. The horizontal dashed dotted lines in panels (b), (d), (f) and (i), delimit the different regions where SPP and SPP modes exist for the TM polarization. The upper panels correspond to the case of two InSb semi-infinite plates, whereas the lower ones are for a multilayer system comprising of $N=32$ unit cells of $\mathrm{InSb} / \mathrm{BK} 7$, with $t_{m}=t_{d}=10 \mathrm{~nm}$, as in Fig. 22(d).

Fig. 5 we present the gap dependence of the ratio between the zero-field heat transfer coefficient and the corresponding coefficient at a given magnitude of the magnetic field for three different values of InSb layer thickness $t_{m}$ and $N=16$. Panels (a-c) display results for the case in which the dielectric layer is made of BK7 glass, while panel (d) shows an example in which this layer is made of $\mathrm{SiO}_{2}$. The first thing to notice is that the applied magnetic field always reduces the heat flux (i.e., $\left.h_{N}(H=0) / h_{N}(H)>1\right)$, irrespective of the dielectric material or the values of the geometrical parameter. Notice also that this field-induced reduction is much more pronounced in the near-field regime. As explained in the introduction, this heat transfer reduction is indeed the expected result from our previous studies of the case of two InSb parallel plates [32 and a plate of InSb separated by a vacuum gap from a InSb thin film 37. These results are clearly at variance with those reported in Ref. 40. that were obtained with the help of an effective medium theory. It is worth stressing that we have found very similar results for different numbers of unit cells (not shown here).

The results presented in Fig. 5 show that the most drastic NFRHT reduction induced by the magnetic field occurs for the thinnest InSb layer. For example, we see in panel (a) that for a $5 \mathrm{~nm}$-thick InSb layer the NFRHT is reduced by up to a $573 \%$ for a gap size of $14.4 \mathrm{~nm}$ and a field magnitude of $8 \mathrm{~T}$. As the InSb layer gets thicker, for example $10 \mathrm{~nm}$ as in panel (b), the maximum reduction becomes on the order of $\sim 500 \%$, and for a thickness of the InSb layer of $100 \mathrm{~nm}$, see panel (c), one essentially recovers the limiting case of two infinite plates discussed in Ref. [32. The reason for this behavior is easy to understand in view of our previous studies [32, 37. For thin InSb layers, the number of these layers whose evanescent field reaches the vacuum gap is larger and, therefore, the replacement of SPPs and SPhPs for modes by hyperbolic ones has a more drastic impact.

A natural question that arises at this point is if the polarization conversion plays any role in the decease of the NFRHT when the magnetic field is applied. To answer this question we have computed the heat transfer in all the examples discussed above, but this time assuming that the the amplitudes $r_{i j}^{p, s}$ and $r_{i j}^{s, p}$ are zero. Let us recall that these off-diagonal reflection coefficients, see Eq. (6), are responsible for the polarization conversion in this system. The results obtained with this approximation are shown in Fig. 5 as circles. As it is evident from these results, the polarization conversion plays no role in the reduction of the NFRHT induced by the external magnetic field. As discussed in our previous work [32, 37, this reduction is due to the fact that the field 

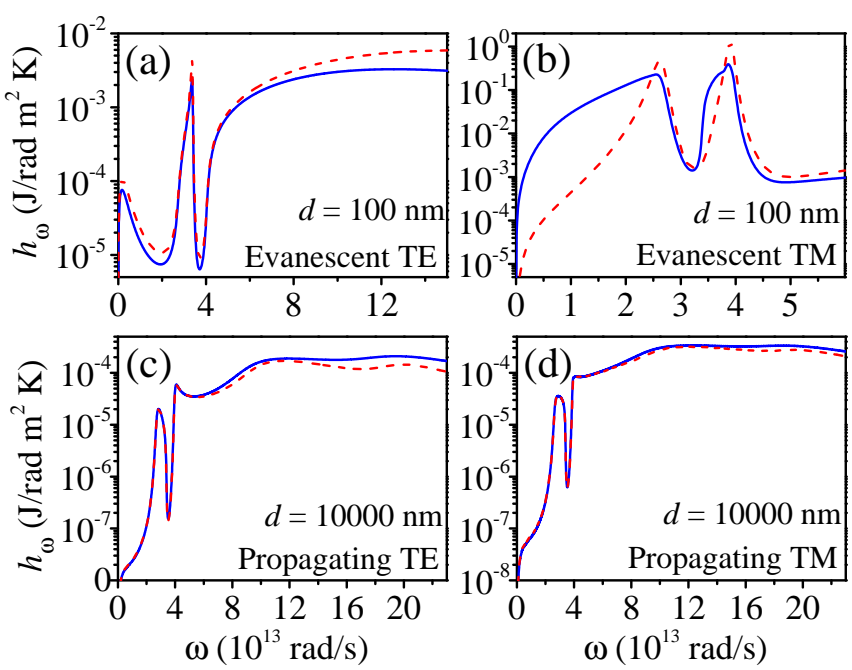

FIG. 4. Comparison between spectral heat transfer coefficients of two InSb infinite plates (dashed lines) and the corresponding one for the two multilayers of Fig. 1(a) (solid lines), with $N=32 \mathrm{InSb} / \mathrm{BK} 7$ unit cells, and $t_{m}=t_{d}=10 \mathrm{~nm}$, as in Fig. 2(d). Panels (a), (b), (c) and (d) correspond, respectively, to the pair of panels [(a) and (e)], [(b) and (f)], [(c) and $(\mathrm{g})]$ and $[(\mathrm{d})$ and $(\mathrm{i})]$, in Fig. 3 .

induces the appearance of hyperbolic modes that progressively replace the zero-field surface modes and they are less effective transferring the heat. A way to confirm this argument is by investigating the spectral heat transfer coefficient, which we show in Fig. 6 for the case of a gap size of $10 \mathrm{~nm}$ shown in Fig. 5 (b) and values of the field equal to $0,2,4$, and $6 \mathrm{~T}$. In this figure we also show the contribution of the TM evanescent waves and the results for the total heat transfer coefficient computed with the diagonal approximation $r_{i j}^{p, s}=r_{i j}^{s, p}=0$. The first thing to notice from these spectral functions is that, as we showed in our previous papers [32, 37, the reduction of the NFRHT is dominated exclusively by the TM polarized evanescent modes, which are suppressed by the emerging hyperbolic modes as the field is cranked up. The second thing is that the results obtained with the diagonal approximation reproduce very accurately the exact results, showing again that the polarization conversion is not relevant for the field-induced NFRHT reduction.

Another interesting observation is the fact that the NFRHT reduction induced by the field is more prominent in the case in which the dielectric layer is made of BK7 glass, which is evident when comparing panels (b) and (d) of Fig. 5 that were obtained for the same value of the dielectric layer thickness. In particular, we find that for an applied field with $8 \mathrm{~T}$ and a gap size of 10 $\mathrm{nm}$, the reduction for the BK7 case is about $488 \%$, while it is $381 \%$ for $\mathrm{SiO}_{2}$. This difference can be understood by examining the evolution of the transmission probability of the TM evanescent modes as the magnitude of the external field varies, see Fig. 7. In the structure with
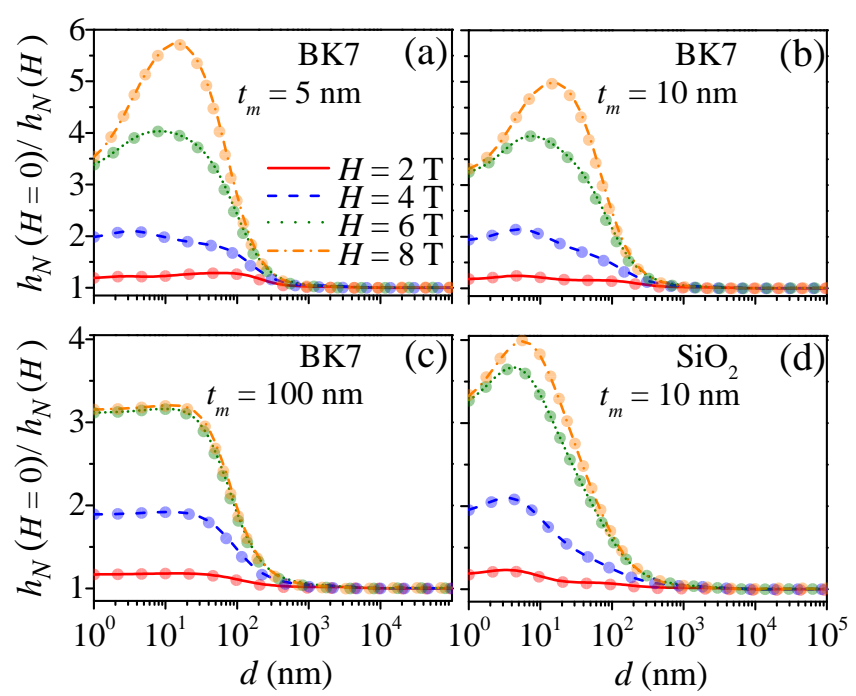

FIG. 5. Ratio between the zero-field heat transfer coefficient and the coefficient at a given value of the field magnitude as a function of the gap size and thickness, $t_{m}$, of the InSb layers. Panels (a)-(c) correspond to an InSb/BK7 unit cell, whereas panel (d) corresponds to an $\mathrm{InSb} / \mathrm{SiO}_{2}$ unit cell. All results were obtained for 16 unit cells and for dielectric layers (either BK7 or $\mathrm{SiO}_{2}$ ) with thickness $t_{d}=10 \mathrm{~nm}$. Circles in panels (a)-(c) correspond to the results obtained with the diagonal approximation $r_{i j}^{s, p}=r_{i j}^{p, s}=0$.

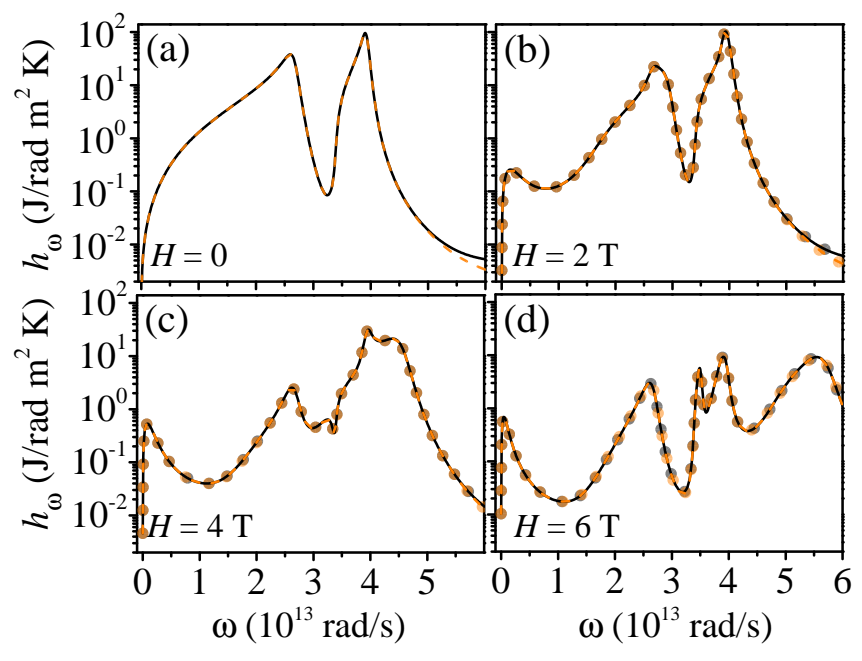

FIG. 6. Total spectral heat transfer coefficient (black solid lines) and the contribution of TM evanescent waves (dashed orange lines) for the same geometrical parameters as in Fig. 5(b) and a gap size of $10 \mathrm{~nm}$. The different panels correspond to different values of the external magnetic field: (a) $0 \mathrm{~T}$, (b) $2 \mathrm{~T}$, (c) $4 \mathrm{~T}$, and (d) $6 \mathrm{~T}$. The number of unit cells in all cases shown in this figure were set to $N=16$. The circles in panels (b)-(d) correspond to the results for the total spectral heat transfer coefficient obtained with the diagonal approximation $r_{i j}^{s, p}=r_{i j}^{p, s}=0$.

InSb/BK7 unit cells, the absence of dispersion in the dielectric function of the BK7 layers makes that SPPs 

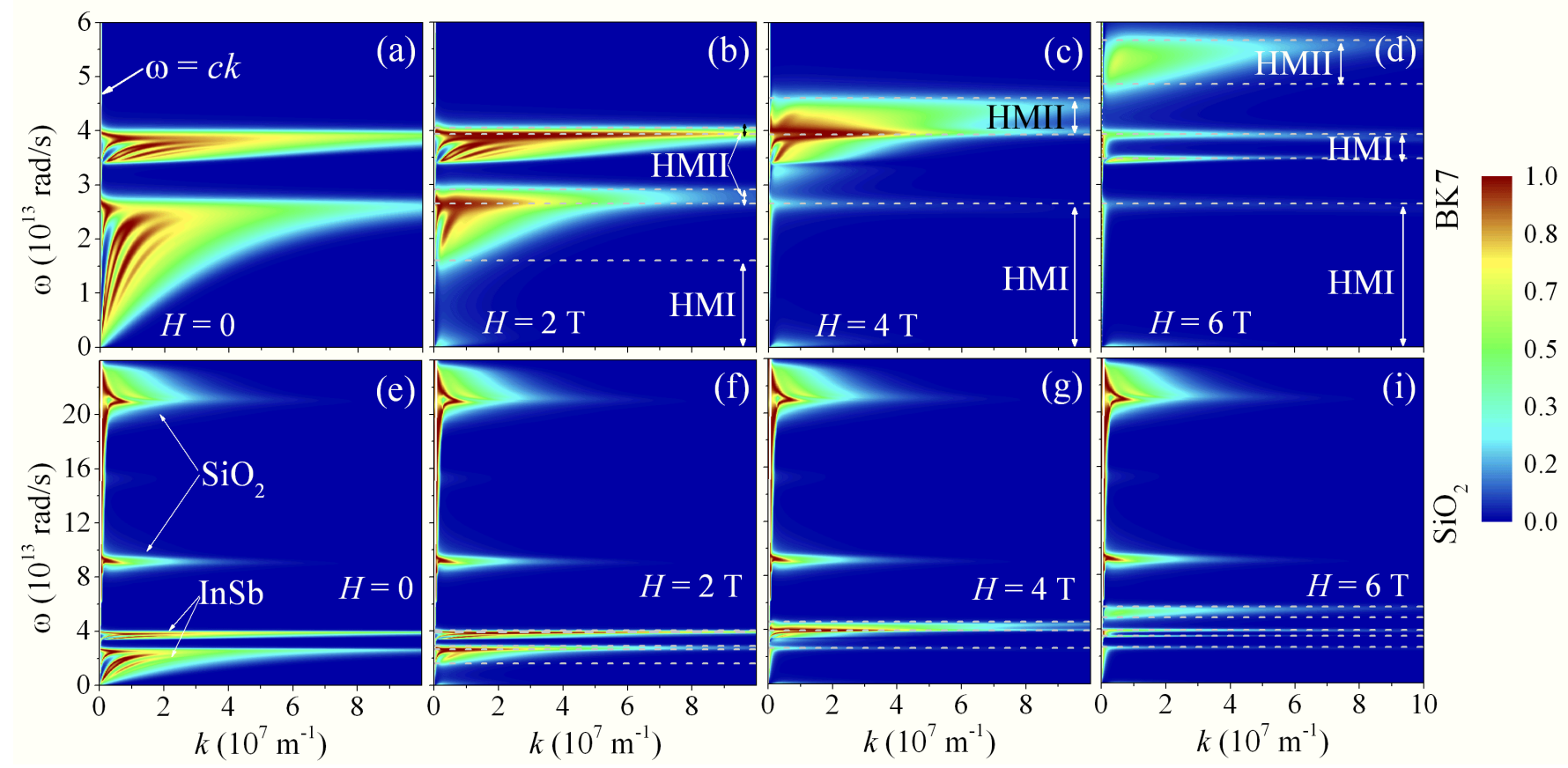

FIG. 7. Transmission coefficient for $p$-polarized (TM) waves as a function of the magnitude of the parallel wave vector and frequency for different magnitudes of the applied magnetic field. Upper (lower) panels correspond to an $\mathrm{InSb}_{\mathrm{BK}} \mathrm{B}\left(\mathrm{InSb} / \mathrm{SiO}{ }_{2}\right.$ ) unit cell. In all panels we have set $t_{m}=t_{d}=d=10 \mathrm{~nm}$. The number of units cells was set to $N=16$.

and $\mathrm{SPhPs}$ modes of InSb be the only ones available to hybridize with the Bloch modes [see panel (a)]. These modes are progressively replaced by hyperbolic modes as the external field increases 32, leading to the reduction of the NFRHT [see panels (b)-(d)]. In contrast, when the dielectric layers are made of a polar material like $\mathrm{SiO}_{2}$, the zero-field transmission probability exhibits, in addition to the maxima related to the SPPs and SPhPs of the InSb layers, other two maxima at higher frequencies related the $\mathrm{SPhPs}$ in the $\mathrm{SiO}_{2}$ layers [see Fig. 11(d)]. So, although the external field replaces the SPP and SPhP modes of InSb by hyperbolic modes, this field cannot modify the SPhP modes due to $\mathrm{SiO}_{2}$ [see panels (f) to (i)], making the structure containing this polar dielectric less sensitive to the application of an external magnetic field.

\section{CONCLUSIONS}

Motivated by the current interest in the active control of NFRHT, we have presented in this paper a systematic theoretical study of the influence of an external magnetic field in the radiative heat exchange between two periodic 1D magneto-photonic crystals. We have analyzed in detail both the zero- and the finite-field radiative heat flux. In the first case, we have shown that for the smallest gaps in the near-field regime, the heat flux between the multilayered structures is higher than for the case of two InSb infinite parallel plates. In agreement with related studies, we find that this enhancement is due to the hybridization of SPP and SPhP modes with the Bloch modes resulting from the translation symmetry in a periodic structure. However, in an intermediate regime for gap sizes between 80 and $2200 \mathrm{~nm}$, the radiative heat transfer is actually smaller in the case of the 1D crystals. We have shown that this is a consequence of a decrease in the efficiency of frustrated TE modes by total internal reflection, and smaller parallel wave vectors for surface TM waves. In contrast, for gap sizes beyond $2200 \mathrm{~nm}$ and, in particular in the far-field regime, there is an increase in the radiative heat flux due to enhanced Fabry-Pérot resonances between and inside multiple layers of each stack.

On the other hand, we have shown that an applied static magnetic field induces the replacement of surface polariton states, which have very high values of the parallel wave vector, by hyperbolic modes with lower parallel wave vectors. The field-induced appearance of these hyperbolic modes leads to a reduction of the NFRHT that is even more pronounced than in the case of two InSb infinite parallel plates. In particular, we have found reduction factors as high as 6 for fields of a few Teslas, which is truly remarkable. Moreover, we have shown that this reduction of the NFRHT is due to the appearance of hyperbolic modes induced by the field and that the polarization conversion in this system plays no role in this effect. Overall, our work provides one more example of the promising possibility to control NFRHT by means of an external magnetic field, and we hope that it will motivate the realization of experiments to confirm these 
interesting thermomagnetic effects.

\section{ACKNOWLEDGMENTS}

J.C.C. acknowledges funding from the Spanish Ministry of Economy and Competitiveness (MINECO) (con- tract No. FIS2017-84057-P).
[1] B. Song, A. Fiorino, E. Meyhofer, and P. Reddy, Nearfield radiative thermal transport: From theory to experiment, AIP Advances 5, 053503 (2015).

[2] J. C. Cuevas and F. J. García-Vidal, Radiative heat transfer, ACS Photonics 5, 3896 (2018).

[3] S.-A. Biehs, R. Messina, P. S. Venkataram, A. W. Rodriguez, J. C. Cuevas, P. Ben-Abdallah, Nearfield radiative heat transfer in many-body systems, arXiv:2007.05604

[4] D. Polder and M. Van Hove, Theory of radiative heat transfer between closely spaced bodies Phys. Rev. B 4, 3303 (1971).

[5] A. Kittel, W. Müller-Hirsch, J. Parisi, S.-A. Biehs, D. Reddig, and M. Holthaus, Near-Field Heat Transfer in a Scanning Thermal Microscope, Phys. Rev. Lett. 95, 224301 (2005).

[6] A. Narayanaswamy, S. Shen, and G. Chen, Near-field radiative heat transfer between a sphere and a substrate, Phys. Rev. B 78, 115303 (2008).

[7] L. Hu, A. Narayanaswamy, X. Y. Chen, and G. Chen, Near-field thermal radiation between two closely spaced glass plates exceeding Planck's blackbody radiation law, Appl. Phys. Lett. 92, 133106 (2008).

[8] E. Rousseau, A. Siria, G. Jourdan, S. Volz, F. Comin, J. Chevrier, and J.-J, Greffet, Radiative heat transfer at the nanoscale, Nat. Photonics 3, 514 (2009).

[9] S. Shen, A. Narayanaswamy, and G. Chen, Surface phonon polaritons mediated energy transfer between nanoscale gaps, Nano Lett. 9, 2909 (2009).

[10] R. S. Ottens, V. Quetschke, S. Wise, A. A. Alemi, R. Lundock, G. Mueller, D. H. Reitze, D. B. Tanner, and B. F. Whiting, Near- Field Radiative Heat Transfer Between Macroscopic Planar Surfaces, Phys. Rev. Lett. 107, 014301 (2011).

[11] S. Shen, A. Mavrokefalos, P. Sambegoro, and G. Chen, Nanoscale thermal radiation between two gold surfaces, Appl. Phys. Lett. 100, 233114 (2012).

[12] T. Kralik, P. Hanzelka, M. Zobac, V. Musilova, T. Fort, and M. Horak, Strong Near-Field Enhancement of Radiative Heat Transfer Between Metallic Surfaces, Phys. Rev. Lett. 109, 224302 (2012).

[13] P. J. van Zwol, L. Ranno, and J. Chevrier, Tuning Near Field Radiative Heat Flux Through Surface Excitations with a Metal Insulator Transition, Phys. Rev. Lett. 108, 234301 (2012).

[14] P. J. van Zwol, S. Thiele, C. Berger, W. A. de Heer, and J. Chevrier, Nanoscale Radiative Heat Flow Due to Surface Plasmons in Graphene and Doped Silicon, Phys. Rev. Lett. 109, 264301 (2012).

[15] B. Guha, C. Otey, C. B. Poitras, S. H. Fan, and M. Lipson, Near-field radiative cooling of nanostructures, Nano Lett. 12, 4546 (2012).
[16] J. Shi, P. Li, B. Liu, and S. Shen, Tuning near field radiation by doped silicon, Appl. Phys. Lett. 102, 183114 (2013).

[17] L. Worbes, D. Hellmann, and A. Kittel, Enhanced nearfield heat flow of a monolayer dielectric island, Phys. Rev. Lett. 110, 134302 (2013).

[18] R. St-Gelais, B. Guha, L. X. Zhu, S. H. Fan, and M. Lipson, Demonstration of strong near-field radiative heat transfer between integrated nanostructures, Nano Lett. 14, 6971 (2014).

[19] B. Song, Y. Ganjeh, S. Sadat, D. Thompson, A. Fiorino, V. Fernández-Hurtado, J. Feist, F. J. Garcia-Vidal, J. C. Cuevas, P. Reddy, and E. Meyhofer, Enhancement of near-field radiative heat transfer using polar dielectric thin films, Nat. Nanotechnol. 10, 253 (2015).

[20] K. Kim, B. Song, V. Fernández-Hurtado, W. Lee, W. Jeong, L. Cui, D. Thompson, J. Feist, M. T. H. Reid, F. J. García-Vidal, J. C. Cuevas, E. Meyhofer, P. Reddy, Radiative heat transfer in the extreme near field, Nature (London) 528, 387 (2015).

[21] M. Lim, S. S. Lee, and B. J. Lee, Near-field thermal radiation between doped silicon plates at nanoscale gaps, Phys. Rev. B 91, 195136 (2015).

[22] R. St-Gelais, L. Zhu, S. Fan, and M. Lipson, Near-field radiative heat transfer between parallel structures in the deep subwavelength regime, Nat. Nanotechnol. 11, 515 (2016).

[23] B. Song, D. Thompson, A. Fiorino, Y. Ganjeh, P. Reddy, E. Meyhofer, Radiative heat conductances between dielectric and metallic parallel plates with nanoscale gaps, Nat. Nanotechnol. 11, 509 (2016).

[24] M. P. Bernardi, D. Milovich, M. Francoeur, Radiative heat transfer exceeding the blackbody limit between macroscale planar surfaces separated by a nanosize vacuum gap, Nat. Commun. 7, 12900 (2016).

[25] L. Cui, W. Jeong, V. Fernández-Hurtado, J. Feist, F. J. García-Vidal, J. C. Cuevas, E. Meyhofer, P. Reddy, Study of radiative heat transfer in Ångström- and nanometre-sized gaps, Nat. Commun. 8, 14479 (2017).

[26] K. Kloppstech, N. Könne, S.-A. Biehs, A. W. Rodriguez, L. Worbes, D. Hellmann, A. Kittel, Giant heat transfer in the crossover regime between conduction and radiation, Nat. Commun. 8, 14475 (2018).

[27] M. Ghashami, H. Geng, T. Kim, N. Iacopino, S.-K. Cho, K. Park, Precision Measurement of Phonon-Polaritonic Near-Field Energy Transfer Between Macroscale Planar Structures Under Large Thermal Gradients, Phys. Rev. Lett. 120, 175901 (2018).

[28] A. Fiorino, D. Thompson, L. Zhu, B. Song, P. Reddy, E. Meyhofer, Giant enhancement in radiative heat transfer in sub-30 nm gaps of plane parallel surfaces, Nano Lett. 18, 3711 (2018). 
[29] J. DeSutter, L. Tang, and M. Francoeur, A near-field radiative heat transfer device, Nat. Nanotechnol. 14, 751 (2019).

[30] S. M. Rytov, Theory of Electric Fluctuations and Thermal Radiation, (Air Force Cambrige Research Center, Bedford, MA, 1953).

[31] S. M. Rytov, Y. A. Kravtsov, and V. I. Tatarskii, Principles of Statistical Radiophysics, Vol. 3 (Springer-Verlag, Berlin Heidelberg, 1989).

[32] E. Moncada-Villa, V. Fernández-Hurtado, F. J. GarcíaVidal, A. García-Martín, and J. C. Cuevas, Magnetic field control of near-field radiative heat transfer and the realization of highly tunable hyperbolic thermal emitters, Phys. Rev B 92, 125418 (2015).

[33] P. Ben-Abdallah, Photon Thermal Hall Effect, Phys. Rev. Lett. 116, 084301 (2016).

[34] L. Zhu and S. Fan, Persistent Directional Current at Equilibrium in Nonreciprocal Many-Body Near Field Electromagnetic Heat Transfer, Phys. Rev. Lett. 117, 134303 (2016).

[35] I. Latella and P. Ben-Abdallah, Giant Thermal Magnetoresis- tance in Plasmonic Structures, Phys. Rev. Lett. 118, 173902 (2017).

[36] R. M. Abraham Ekeroth, P. Ben-Abdallah, J. C. Cuevas, and García-Martín, Anisotropic thermal magnetoresistance for an active control of radiative heat transfer, ACS Photonics 5, 705 (2018).

[37] E. Moncada-Villa and J. C. Cuevas, Magnetic field effects in the near-field radiative heat transfer between planar structures, Phys. Rev. B 101, 085411 (2020).

[38] A. Ott, R. Messina, P. Ben-Abdallah, and S.-A. Biehs, Magnetothermoplasmonics: from theory to applications, J. Photon. Energy 9, 032711 (2019).

[39] E. Moncada-Villa, A. I. Fernández-Domínguez, J. C. Cuevas, Magnetic-field controlled anomalous refraction in doped semiconductors, J. Opt. Soc. Am. B 36, 935 (2019).

[40] J. Song, Q. Cheng, L. Lu, B. Li, K. Zhou, B. Zhang, Z. Luo, and X. Zhou, Magnetically tunable near-field radiative heat transfer in hyperbolic metamaterials, Phys. Rev. Appl. 13024054 (2020).

[41] Y. Guo, C. L. Cortes, S. Molesky, and Z. Jacob, Broadband super-Planckian thermal emission from hyperbolic metamaterials, Appl. Phys. Lett. 101, 131106 (2012).

[42] S. A. Biehs, M. Tschikin, and P. Ben-Abdallah, Hyperbolic Metamaterials as an Analog of a Blackbody in the Near Field, Phys. Rev. Lett. 109, 104301 (2012).
[43] Y. Guo and Z. Jacob, Thermal hyperbolic metamaterials, Opt. Express 21, 15014 (2013).

[44] S.-A. Biehs, M. Tschikin, R. Messina, and P. BenAbdallah, Super-Planckian near-field thermal emission with phonon-polaritonic hyperbolic metamaterials, Appl. Phys. Lett. 102, 131106 (2013).

[45] T. J. Bright, X. L. Liu, and Z. M. Zhang, Energy streamlines in near-field radiative heat transfer between hyperbolic metamaterials, Opt. Express 22, A1112 (2014).

[46] O. D. Miller, S. G. Johnson, and A. W. Rodriguez, Effectiveness of Thin Films in Lieu of Hyperbolic Metamaterials in the Near Field, Phys. Rev. Lett. 112, 157402 (2014).

[47] S.-A. Biehs and P. Ben-Abdallah, Near-field heat transfer between multilayer hyperbolic metamaterials, Z. Naturforsch. A 72, 115 (2017).

[48] H. Iizuka and S. Fan, Significant enhancement of nearfield electromagnetic heat transfer in a multilayer structure through multiple surface-states coupling, Phys. Rev. Lett. 120, 063901 (2018).

[49] V. Fernández-Hurtado, F. J. Garcia-Vidal, S. Fan, J. C. Cuevas, Enhancing Near-Field Radiative Heat Transfer with Si-Based Metasurfaces, Phys. Rev. Lett. 118, 203901 (2017).

[50] A. Zvezdin and V. Kotov, Modern Magnetooptics and Magnetooptical Materials, (IOP, Bristol, 1997).

[51] E. D. Palik, R. Kaplan, R. W. Gammon, H. Kaplan, R. F. Wallis, and J. J. Quinn, Coupled surface magnetoplasmon-optic-phonon polariton modes on InSb, Phys. Rev. B 13, 2497 (1976).

[52] E. D. Palik, Handbook of Optical Constants of Solids (Academic Press, London, 1985).

[53] J. P. Mulet, K. Joulain, R. Carminati, and J. J. Greffet, Enhanced radiative heat transfer at nanometric distances, Microscale Therm. Eng. 6, 209 (2002).

[54] S.-A. Biehs, P. Ben-Abdallah, F. S.S. Rosa, K. Joulain, and J.-J. Greffet, Nanoscale heat flux between nanoporous materials, Opt. Express 19, A1088 (2011).

[55] B. Caballero, A. García-Martín, and J. C. Cuevas, Generalized scattering-matrix approach for magneto-optics in periodically patterned multilayer systems, Phys. Rev. B 85, 245103 (2012).

[56] X. L. Liu, T. J. Bright, and Z. M. Zhang, Application conditions of effective medium theory in the near-field radiative heat transfer between multilayered metamaterials, J. Heat Transfer 136, 092703 (2014). 